\title{
On periodic self-homeomorphisms of closed orientable surfaces determined by their orders
}

\author{
C. Bagiński ${ }^{1}$ - M. Carvacho $^{2}$ - G. Gromadzki ${ }^{3}$. \\ R. Hidalgo 4
}

Received: 29 November 2014 / Accepted: 2 August 2015 / Published online: 13 August 2015

(C) The Author(s) 2015. This article is published with open access at Springerlink.com

\begin{abstract}
The fundamentals for the topological classification of periodic orientationpreserving self-homeomorphisms of a closed orientable topological surface $X$ of genus $g \geq 2$ have been established, by Nielsen, in the thirties of the last century. Here we consider two concepts related to this classification; rigidity and weak rigidity. A cyclic action $G$ of order $N$ on $X$ is said to be topologically rigid if any other cyclic action of order $N$ on $X$ is topologically conjugate to it. If this assertion holds for arbitrary other action but having, in addition, the same orbit genus and the same structure of singular orbits, then $G$ is said to be weakly topologically rigid. We give a precise description of rigid and weakly rigid cyclic quasi-platonic actions which mean actions having three singular orbits and for which $X / G$ is a sphere.
\end{abstract}

Keywords Periodic self-homeomorphisms of closed orientable surfaces ·

Their classification · Riemann surfaces · Their automorphisms · (Real) algebraic curves

C. Bagiński and G. Gromadzki supported by NCN 2012/05/B/ST1/02171. G. Gromadzki supported additionally by the Fondecyt 1110001 of Chilean Ministry of Sciences and Max Planck Mathematical Institute in Bonn. R. Hidalgo partially supported by Project Fondecyt 1150003. M. Carvacho supported by CONICYT 79112005 and UTFSM 12.13.72.

\footnotetext{
G. Gromadzki

grom@mat.ug.edu.pl

C. Bagiński

c.baginski@pb.edu.pl

M. Carvacho

mariela.carvacho@usm.cl

R. Hidalgo

ruben.hidalgo@ufrontera.cl

1 Faculty of Computer Science, Białystok University of Technology, Białystok, Poland

2 Departamento de Matemática, UTFSM, Valparaiso, Chile

3 Institute of Mathematics, Gdańsk University, Gdańsk, Poland

4 Departamento de Matemática y Estadística, Universidad de La Frontera, Casilla 54-D, 4780000 Temuco, Chile
} 
Mathematics Subject Classification Primary 30F; Secondary 57M · 14H

\section{Introduction}

The fundamentals for the topological classification of periodic orientation-preserving selfhomeomorphisms of a closed topological surface $X$ of genus $g \geq 2$ have been established, by Nielsen, in the thirties of the last century [8]. Certain classifications have been given also by Yokayama [11] and Nakagawa [7] who proved that a closed Riemann surface of genus $g \neq 4,6,9,10,12$ having an automorphism of order $N \geq 3 g$ is determined (up to birational equivalence) by $N$. More recently, these results have been reproved independently by Hirose [5] using less explicit methods which, however, are closer to our approach in this paper. Actually in this case, there are very few possibilities for such orders; $N=4 g+2,4 g, 3 g$ or $3 g+3$ and for the first three cases such configuration actually exists for arbitrary $g$ while the last one exists if and only if $g \not \equiv 2(\bmod 3)$. The order $N$ of a cyclic action of $G=\mathbb{Z}_{N}$ on $X$ is said to be topologically rigid if for any other cyclic action $G^{\prime}$ of order $N$ on $X, G$ and $G^{\prime}$ are conjugate by a certain orientation-preserving self-homeomorphism of $X$ which does not need to be periodic.

It seems that the above rigidity property, for mentioned orders of cyclic actions, is a rather rare phenomenon and here, apart of it, we consider and study another related property of periodic cyclic actions called weak rigidity. We say that the cyclic action $G$ on $X$ is weakly rigid if any other cyclic action $G^{\prime}$ on $X$ of the same order, with the same orbit genus and lengths structure of singular orbits, is topologically conjugate to it. It is clear that rigidity implies weak rigidity. Further if any action of order $N$ on $X$ is weakly rigid, then $N$ is said to be weakly rigid order. In Theorem 3.9, we use combinatorial techniques to characterize the weakly rigid cyclic actions having three singular orbits and the orbit space $X / G$ of genus zero. These actions are examples of so called quasi-platonic actions which get a great deal of attention in the literature. Observe first that they can be realized as birational actions on complex algebraic curves, due to the geometrization theorem of Nielsen and the Riemann uniformization. Due to the famous theorem of Belyi [1], these curves can be defined over the algebraic numbers. Their equations were found by Wootton in [10] for prime order $N$. They are also building blocks for the construction of Beauville surfaces which are important since they form a natural domain for the action of the absolute Galois group, $\operatorname{Aut}(\overline{\mathbb{Q}} / \mathbb{Q})$. By another result of Singerman [9], Riemann surfaces considered here are symmetric and so they have some real forms. Furthermore, by Köck and Singerman [6] these forms can be defined over the real algebraic numbers. In [3], Bujalance et al. have found the number of such forms and their topological type. This gives us the number of connected components and separability of the smooth projective models for them. Finally in a recent paper [2], Benim and Wootton gave nice theoretical formulae for the number of topologically distinct actions of cyclic groups on quasi-platonic Riemann surfaces. Our result does not seem, however, possible to get from [2], at least in an easy direct way.

\section{Preliminaries}

We shall use the following ingredients. Throughout this paper, $X$ will denote a closed orientable surface of genus $g \geq 2$. 


\subsection{Nielsen's geometrization}

Let $\varphi$ be an orientation-preserving self-homeomorphism of $X$ of finite order $N$. There exists a structure of a Riemann surface on $X$ (we still denote the resulting Riemann surface also by $X$ by abuse of language) so that $\varphi$ is a conformal automorphism. In the rest of the paper, we assume $X$ to have such a Riemann surface structure when necessary.

\subsection{Riemann uniformization theorem and elementary covering theory}

The Riemann uniformization theorem asserts that a closed orientable Riemann surface $X$ of genus $g \geq 2$ is isomorphic to the orbit space $\mathcal{H} / \Gamma$ of the hyperbolic upper half plane $\mathcal{H}$ with the holomorphic structure inherited from $\mathcal{H}$, where $\Gamma$ is a Fuchsian group with signature $(g ;-)$. Such a group $\Gamma$ is algebraically isomorphic to the fundamental group of $X$ and so it has the presentation

$$
\left\langle a_{1}, b_{1}, \ldots, a_{g}, b_{g} \mid\left[a_{1}, b_{1}\right] \ldots\left[a_{g}, b_{g}\right]\right\rangle,
$$

where $[a, b]$ denotes the commutator $a b a^{-1} b^{-1}$. Furthermore, elementary covering theory shows that, having a group $G$ of conformal automorphisms of $X$ so represented, we have an isomorphism $G \cong \Lambda / \Gamma$ for some Fuchsian group $\Lambda$, say with signature $\left(h ; m_{1}, \ldots, m_{r}\right)$, which means that it has the presentation

$$
\left\langle x_{1}, \ldots, x_{r}, a_{1}^{\prime}, b_{1}^{\prime}, \ldots, a_{h}^{\prime}, b_{h}^{\prime} \mid x_{1}^{m_{1}}, \ldots, x_{r}^{m_{r}}, x_{1} \ldots x_{r}\left[a_{1}^{\prime}, b_{1}^{\prime}\right] \ldots\left[a_{h}^{\prime}, b_{h}^{\prime}\right]\right\rangle .
$$

So each group $G$ of conformal automorphisms of a closed Riemann surface $X$ leads to a Fuchsian group $\Lambda$ and an epimorphism $\theta: \Lambda \rightarrow G$ with a torsion-free kernel (which in such case is isomorphic to the fundamental group of $X$ without any extra conditions). Conversely an epimorphism $\theta: \Lambda \rightarrow G$ with a torsion-free kernel $\Gamma$ gives rise to an action of $G$ on $X=\mathcal{H} / \Gamma$ through conformal automorphisms defined by

$$
g x=\pi(\lambda(h))
$$

if $g=\theta(\lambda)$ and $x=\pi(h)$ for the canonical projection $\pi: \mathcal{H} \rightarrow X$. Such an epimorphism will be called a smooth or surface-kernel epimorphism throughout the whole paper.

In this paper, we will mainly be interested in triangle groups, being Fuchsian groups with signatures $\left(0 ; m_{1}, m_{2}, m_{3}\right)$ which will also be abbreviated as $\left(m_{1}, m_{2}, m_{3}\right)$. They have the presentations

$$
\left\langle x_{1}, x_{2}, x_{3} \mid x_{1}^{m_{1}}, x_{2}^{m_{2}}, x_{3}^{m_{3}}, x_{1} x_{2} x_{3}\right\rangle .
$$

Furthermore, the Riemann-Hurwitz formula says in this special situation that

$$
2(g-1)=|G|\left(1-\frac{1}{m_{1}}-\frac{1}{m_{2}}-\frac{1}{m_{3}}\right) .
$$

\subsection{Harvey criterion}

Let $\Lambda$ be a Fuchsian group with signature $\left(h ; m_{1}, \ldots, m_{r}\right)$ and let $M=1 \mathrm{~cm}\left(m_{1}, \ldots, m_{r}\right)$. Then there exists a smooth epimorphism from $\Lambda$ onto $\left\langle x \mid x^{N}\right\rangle$ if and only if

$$
\left\{\begin{array}{l}
\text { (a) } M=\operatorname{lcm}\left(m_{1}, \ldots, m_{i-1}, m_{i+1}, \ldots, m_{r}\right) \text { for all } i \\
\text { (b) } M \text { divides } N \text { and if } h=0 \text { then } M=N \\
\text { (c) } r \neq 1 \text { and if } h=0 \text { then } r \geq 3 \text {; } \\
\text { (d) if } N \text { is even then the number of periods } m_{i} \text { with } N / m_{i} \text { odd is even. }
\end{array}\right.
$$




\subsection{Maclachlan decomposition}

In the case that $h=0, r=3$, condition (a) in (5) above for the signature $\left(m_{1}, m_{2}, m_{3}\right)$ is equivalent to the following canonical decomposition:

$$
\left\{\begin{array}{l}
m_{1}=A A_{2} A_{3}, \\
m_{2}=A A_{1} A_{3}, \\
m_{3}=A A_{1} A_{2},
\end{array}\right.
$$

where $A=\operatorname{gcd}\left(m_{1}, m_{2}, m_{3}\right)$ and $A_{k}=\operatorname{gcd}\left(m_{i}, m_{j}\right) / A$, for $k \neq i, j$. Note that the integers $A_{i}$ are pairwise relatively prime and, by (b) in (5), that $N=A A_{1} A_{2} A_{3}$. Condition (d) in (5), states that $N=A A_{1} A_{2} A_{3}$ even is equivalent to having just one of the $A_{i}$ even. This decomposition has been discovered in [4]. The collection $A, A_{1}, A_{2}, A_{3}$ will be called the Maclachlan decomposition of $\left(m_{1}, m_{2}, m_{3}\right)$. A signature $\left(m_{1}, m_{2}, m_{3}\right)$ satisfying (5) for $h=0, r=3$ will be called admissible or $N$-admissible and a quadruple $\left(A, A_{1}, A_{2}, A_{3}\right)$ uniquely determined by (6) we will be called admissible as well.

\subsection{Few words on topological conjugacy}

Two orientation-preserving self-homeomorphisms $\varphi_{1}, \varphi_{2}$ of a surface $X$ are topologically equivalent if they are conjugate by an orientation-preserving self-homeomorphism $f$ of $X$. We shall use conformal models for these actions described in Sects. 2.1 and 2.2. In the conformal setting, the general definition is that two conformal actions $G_{1}$ and $G_{2}$ on Riemann surfaces $X_{1}$ and $X_{2}$ given by smooth epimorphisms $\theta_{1}: \Lambda_{1} \rightarrow G_{1}$ and $\theta_{2}: \Lambda_{2} \rightarrow G_{2}$ are topologically equivalent if and only if the diagram

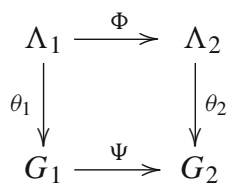

commutes for some isomorphisms $\Phi: \Lambda_{1} \rightarrow \Lambda_{2}, \Psi: G_{1} \rightarrow G_{2}$. The Nielsen isomorphism theorem asserts that $\Phi$ can be chosen to be the conjugation by a self-homeomorphism $f$ of $\mathcal{H}$ and, throughout the paper, we understand that $f$ preserves orientation.

Furthermore, the signature $\left(m_{1}, m_{2}, m_{3}\right)$ is said to be rigid if for any group $\Lambda$ with such signature, any two smooth epimorphism $\theta_{1}, \theta_{2}: \Lambda \rightarrow \mathbb{Z}_{N}$ are equivalent in the above sense. If an $N$-admissible signature is unique, up to ordering of $m_{1}, m_{2}, m_{3}$, and it is rigid, then $N$ is said to be rigid order. If there is more than one $N$-admissible signature but all of them are rigid then the order is said to be weakly rigid. Finally if none of these holds, then the order is simply said to be non-rigid.

\section{Rigid and weakly rigid orders}

Given a positive integer $N$ and a triangle Fuchsian group $\Lambda$ with an $N$-admissible signature $\left(m_{1}, m_{2}, m_{3}\right)$, let

$$
\mathcal{K}=\left\{k<m_{2} \mid \operatorname{gcd}\left(k, m_{2}\right)=1\right\} .
$$

Fix a generator $a$ of $\mathbb{Z}_{N}$ and let $\mathcal{L}$ be the subset of $\mathcal{K}$ consisting of those $k$ for which

$$
a^{N / m_{1}+k N / m_{2}}
$$


has order $m_{3}$. Observe that $K=\varphi\left(m_{2}\right)$, where $\varphi$ is the Euler's totient function, is the cardinality of $\mathcal{K}$ and denote by $L$ be cardinality of $\mathcal{L}$. Then we have the following lemma by which the number of topological equivalency classes of cyclic quasiplatonic actions of type $\left(m_{1}, m_{2}, m_{3}\right)$ is bounded by $L$.

Lemma 3.1 An arbitrary smooth epimorphism $\theta: \Lambda \rightarrow \mathbb{Z}_{N}=\langle a\rangle$ is topologically equivalent to $\theta_{k}$ for some $k \in \mathcal{L}$, where $\theta_{k}\left(x_{1}\right)=a^{N / m_{1}}$ and $\theta_{k}\left(x_{2}\right)=a^{k N / m_{2}}$.

Proof Obviously we have

$$
\theta\left(x_{1}\right)=a^{m N / m_{1}}, \quad \theta\left(x_{2}\right)=a^{k^{\prime} N / m_{2}}, \quad \theta\left(x_{3}\right)=a^{l^{\prime} N / m_{3}},
$$

where $\operatorname{gcd}\left(m, m_{1}\right)=\operatorname{gcd}\left(k^{\prime}, m_{2}\right)=\operatorname{gcd}\left(l^{\prime}, m_{3}\right)=1$, for $\theta$ to preserve the orders of $x_{1}, x_{2}$, and $x_{3}$, and

$$
m \frac{N}{m_{1}}+k^{\prime} \frac{N}{m_{2}}+l^{\prime} \frac{N}{m_{3}} \equiv 0 \quad(\bmod N),
$$

to guarantee $\theta\left(x_{1} x_{2} x_{3}\right)=1$. Now let $m^{\prime}$ be the inversion of $m$ modulo $m_{1}$. Let also $A_{1}^{\prime}$ be the maximal divisor of $A_{1}=N / m_{1}$ coprime to $m_{1}$. Then by the Chinese Remainder Theorem there exists $\alpha$ such that $\alpha \equiv m^{\prime}\left(\bmod m_{1}\right)$ and $\alpha \equiv 1\left(\bmod A_{1}^{\prime}\right)$. Then $\operatorname{gcd}(\alpha, N)=1$ and

$$
\left(a^{m N / m_{1}}\right)^{\alpha}=\left(a^{N / m_{1}}\right)^{m \alpha}=a^{N / m_{1}} .
$$

So $k=k^{\prime} \alpha \in \mathcal{L}$ and for $\Phi=\operatorname{id}_{\Lambda}$ and $\Psi(a)=a^{\alpha}$ in (7), we obtain that $\theta$ is topologically equivalent to $\theta_{k}$ defined by

$$
\theta_{k}\left(x_{1}\right)=a^{N / m_{1}}, \quad \theta_{k}\left(x_{2}\right)=a^{k N / m_{2}}, \quad \theta_{k}\left(x_{3}\right)=a^{l N / m_{3}},
$$

where $l=l^{\prime} \alpha$.

The next lemma will be used later, to decide when two smooth epimorphisms $\theta_{k_{1}}$ and $\theta_{k_{2}}$ from the Lemma 3.1 define topologically equivalent actions.

Lemma 3.2 Let $\mathcal{S}=\operatorname{Stab}_{\mathbb{Z}_{N}^{*}}\left(a^{N / m_{1}}\right)$ be the stabilizer of $a^{N / m_{1}}$ in the group $\operatorname{Aut}(\langle a\rangle)$ and let $S$ be its cardinality. Then $S=\varphi(N) / \varphi\left(m_{1}\right)$.

Proof Indeed

$$
\begin{aligned}
\varphi\left(m_{1}\right) & =\left|\operatorname{Orb}_{\mathbb{Z}_{N}^{*}}\left(a^{N / m_{1}}\right)\right| \\
& =\left[\mathbb{Z}_{N}^{*}: \operatorname{Stab}_{\mathbb{Z}_{N}^{*}}\left(a^{N / m_{1}}\right)\right] \\
& =\frac{\varphi(N)}{\left|\operatorname{Stab}_{\mathbb{Z}_{N}^{*}}\left(a^{N / m_{1}}\right)\right|}
\end{aligned}
$$

and so the assertion.

Lemma 3.3 Each element of $\mathcal{S}$ acts on $\mathcal{K}$ without fixed points. That is, the group $\mathcal{S}$ acts faithfully on $\mathcal{K}$.

Proof Let us take $k \in \mathcal{K}$ and $s, s^{\prime} \in \mathcal{S}$. Recall that these mean $\operatorname{gcd}\left(k, m_{2}\right)=1$ and $\operatorname{gcd}\left(s^{\prime}, N\right)=\operatorname{gcd}(s, N)=1$. Furthermore, from the definition of $\mathcal{S}$ we have $\left(a^{N / m_{1}}\right)^{s}=$ $a^{N / m_{1}}$ which in turn gives $N(s-1) / m_{1} \equiv 0(\bmod N)$. This means in particular that $m_{1}$ 
divides $s-1$. Similarly we can show that $m_{1}$ divides $s^{\prime}-1$. Consequently $m_{1}$ divides $s-s^{\prime}$. Suppose that

$$
\left(a^{N k / m_{2}}\right)^{s}=\left(a^{N k / m_{2}}\right)^{s^{\prime}}
$$

Then

$$
N \frac{k\left(s-s^{\prime}\right)}{m_{2}} \equiv 0 \quad(\bmod N)
$$

We see that $m_{2}$ divides $k\left(s-s^{\prime}\right)$ and therefore $m_{2}$ divides $s-s^{\prime}$ which give $s \equiv s^{\prime}$ $(\bmod N)$.

Now we shall look for $L$. The decomposition from (6) will be crucial here. We have

$$
N=\operatorname{lcm}\left(m_{1}, m_{2}, m_{3}\right)=\operatorname{lcm}\left(m_{1}, m_{2}\right)=\operatorname{lcm}\left(m_{1}, m_{3}\right)=\operatorname{lcm}\left(m_{2}, m_{3}\right),
$$

where $N=A A_{1} A_{2} A_{3}, N / m_{i}=A_{i}$. Furthermore we know by (5) that if $N$ is even, then the number of those $A_{i}$ which are odd is even and therefore, since all $A_{i}$ can not be even, only one of them is even. With these notations, the elements of the form (8) can be written as $a^{A_{1}+k A_{2}}$ and so the set $\mathcal{L}$ can be seen as

$$
\mathcal{L}=\left\{k<A A_{1} A_{3} \mid \operatorname{gcd}\left(A_{1}+k A_{2}, N\right)=A_{3}, \operatorname{gcd}\left(k, A A_{1} A_{3}\right)=1\right\} .
$$

To state the next theorem, we define $\varphi^{\prime}(1)=1$ and for given a prime decomposition $n=$ $p_{1}^{\alpha_{1}} \ldots p_{r}^{\alpha_{r}}>1$ we put

$$
\varphi^{\prime}(n)=\prod_{i=1}^{r}\left(p_{i}-2\right) p_{i}^{\alpha_{i}-1} .
$$

Remark 3.4 Observe the analogy of the function $\varphi^{\prime}$ defined by (11) with the classical Euler's totient function $\varphi$, both in this what concern its algebraic properties and the explicit formula. We see that both of them can be seen as particular cases of function

$$
\varphi_{k}(n)=\prod_{i=1}^{r}\left(p_{i}-k\right) p_{i}^{\alpha_{i}-1}
$$

which can be defined for arbitrary $k$. Namely we have $\varphi=\varphi_{1}$ and $\varphi^{\prime}=\varphi_{2}$.

Lemma 3.5 Let $D$ be the largest divisor of $A$ coprime with $A_{1} A_{2} A_{3}$ and let $B=A / D$. Then

$$
L=\varphi\left(A_{1} B\right) \varphi^{\prime}(D) .
$$

Proof Let $D=p_{1}^{\delta_{1}} \ldots p_{d}^{\delta_{d}}$ and $N=q_{11}^{\alpha_{1}} \ldots q_{1 a}^{\alpha_{a}} q_{21}^{\beta_{1}} \ldots q_{2 b}^{\beta_{b}} q_{31}^{\gamma_{1}} \ldots q_{3 c}^{\gamma_{c}} D$, for different primes $p_{i}$ and $q_{i j}$. Then

$$
A_{1}=q_{11}^{\alpha_{1}^{\prime}} \ldots q_{1 a}^{\alpha_{a}^{\prime}}, A_{2}=q_{21}^{\beta_{1}^{\prime}} \ldots q_{2 b}^{\beta_{b}^{\prime}}, A_{3}=q_{31}^{\gamma_{1}^{\prime}} \ldots q_{3 c}^{\gamma_{c}^{\prime}}
$$

for some $\alpha_{i}^{\prime}, \beta_{i}^{\prime}, \gamma_{i}^{\prime}$ in ranges $0<\alpha_{i}^{\prime} \leq \alpha_{i}, 0<\beta_{i}^{\prime} \leq \beta_{i}, 0<\gamma_{i}^{\prime} \leq \gamma_{i}$. Let

$$
B_{1}=q_{11}^{\alpha_{1}-\alpha_{1}^{\prime}} \ldots q_{1 a}^{\alpha_{a}-\alpha_{a}^{\prime}}, \quad B_{2}=q_{21}^{\beta_{1}-\beta_{1}^{\prime}} \ldots q_{2 b}^{\beta_{b}-\beta_{b}^{\prime}}, \quad B_{3}=q_{31}^{\gamma_{1}-\gamma_{1}^{\prime}} \ldots q_{3 c}^{\gamma_{c}-\gamma_{c}^{\prime}} .
$$

Then $B=B_{1} B_{2} B_{3}$ and $D$ together with

$$
A_{1} B_{1}=q_{11}^{\alpha_{1}} \ldots q_{1 a}^{\alpha_{a}}, \quad A_{2} B_{2}=q_{21}^{\beta_{1}} \ldots q_{2 b}^{\beta_{b}}, \quad A_{3} B_{3}=q_{31}^{\gamma_{1}} \ldots q_{3 c}^{\gamma_{c}}
$$


are all pairwise coprime. Therefore

$$
\mathbb{Z}_{N} \cong \mathbb{Z}_{A_{1} B_{1}} \oplus \mathbb{Z}_{A_{2} B_{2}} \oplus \mathbb{Z}_{A_{3} B_{3}} \oplus \mathbb{Z}_{p_{1}^{\delta_{1}}} \oplus \cdots \oplus \mathbb{Z}_{p_{r}^{\delta_{d}}}
$$

and

$$
\mathbb{Z}_{A A_{1} A_{3}} \cong \mathbb{Z}_{A_{1} B_{1}} \oplus \mathbb{Z}_{B_{2}} \oplus \mathbb{Z}_{A_{3} B_{3}} \oplus \mathbb{Z}_{p_{1}^{\delta_{1}}} \oplus \cdots \oplus \mathbb{Z}_{p_{r}^{\delta_{d}}} .
$$

Hence every element $x \in \mathbb{Z}_{A A_{1} A_{3}}$ can be represented as a sequence

$$
\left(x_{1}, x_{2}, x_{3}, x_{1}^{\prime}, \ldots, x_{r}^{\prime}\right)
$$

with $x_{1} \in \mathbb{Z}_{A_{1} B_{1}}, x_{2} \in \mathbb{Z}_{B_{2}}, x_{3} \in \mathbb{Z}_{A_{3} B_{3}}$ and $x_{j}^{\prime} \in \mathbb{Z}_{p_{j}^{\delta_{j}}}$. Similarly the elements of $\mathbb{Z}_{N}$ are represented. Moreover if $x$ is invertible in $\mathbb{Z}_{A A_{1} A_{3}}^{*}$ then all components of the sequence corresponding to $x$ are invertible in suitable rings.

Now, for $x \in \mathbb{Z}_{A A_{1} A_{3}}$, the correspondence $x \rightarrow A_{1}+x A_{2}(\bmod N)$ is an injection from $\mathbb{Z}_{A A_{1} A_{3}}$ to $\mathbb{Z}_{N}$. In fact, if $A_{1}+x A_{2} \equiv A_{1}+x^{\prime} A_{2}(\bmod N)$, then $N$ divides $A_{2}\left(x-x^{\prime}\right)$ which in turn means that $A A_{1} A_{3}$ divides $x-x^{\prime}$.

Let $\left(A_{1}+x_{1} A_{2}, A_{1}+x_{2} A_{2}, A_{1}+x_{3} A_{2}, A_{1}+x_{1}^{\prime} A_{2}, \ldots, A_{1}+x_{r}^{\prime} A_{2}\right)$ be the sequence corresponding to $A_{1}+x A_{2}$ with entries taken modulo a suitable number. If we assume that $x \in \mathbb{Z}_{A A_{1} A_{3}}^{*}$, we need to know how many images of the function satisfy the condition $\operatorname{gcd}\left(A_{1}+x A_{2}, N\right)=A_{3}$. That is how many corresponding sequences of $A_{1}+x A_{2}$ for invertible $x \in \mathbb{Z}_{A A_{1} A_{3}}$ have all components invertible beside the third one, which has to be of the form $A_{1}+x_{3} A_{2}=t A_{3}$, where $\operatorname{gcd}\left(t, B_{3}\right)=1$. (Note that if $\operatorname{gcd}\left(t, B_{3}\right)=t_{1}>1$, then $\operatorname{gcd}\left(A_{1}+x A_{2}, N\right)=t_{1} A_{3}>A_{3}$. $)$

For counting all possible values of $x$ satisfying the above, we determine the numbers of possible choices of $x_{i}$ 's corresponding to $x$. Note first that for $x \in \mathbb{Z}_{A A_{1} A_{3}}^{*}$ all possible values of $t$ satisfying $A_{1}+x_{3} A_{2}=t A_{3}$ are achievable. Actually, the condition $A_{1}+x_{3} A_{2} \equiv t A_{3}$ $\left(\bmod A_{3} B_{3}\right)$ means $A_{2} x_{3} \equiv\left(t A_{3}-A_{1}\right)\left(\bmod A_{3} B_{3}\right)$. Since $t A_{3}-A_{1}$ and $A_{2}$ are relatively prime to $A_{3} B_{3}$, there exist such invertible $x_{3}$ in $\mathbb{Z}_{A_{3} B_{3}}$. That is, there are $\varphi\left(B_{3}\right)$ choices for $t$ and therefore $\varphi\left(B_{3}\right)$ choices for $x_{3}$.

Next, for $x \in \mathbb{Z}_{A A_{1} A_{3}}^{*}$ we have $\operatorname{gcd}\left(A_{1}+x A_{2}, A_{1} B_{1} A_{2} B_{2}\right)=1$, so for a fixed $t$ satisfying $A_{1}+x A_{2}=t A_{3}, \operatorname{gcd}\left(t, B_{3}\right)=1$, we have $\varphi\left(A_{1} B_{1}\right) \varphi\left(B_{2}\right)$ possibilities for the first two entries of the sequence.

Finally, let us consider $A_{1}+x_{j}^{\prime} A_{2}$. Write $x_{j}^{\prime}=y_{j}+p_{j} z_{j}$, where $1 \leq y_{j} \leq p_{j}-1$ and $0 \leq z_{j} \leq p_{j}^{\delta_{j}-1}-1$. Then $A_{1}+x_{j}^{\prime} A_{2}=\left(A_{1}+y_{j} A_{2}\right)+p_{j} z_{1} A_{2}$ and for exactly one value of $y_{j}$ we have $A_{1}+y_{j} A_{2} \equiv 0\left(\bmod p_{j}\right)$. Therefore we have $\left(p_{j}-2\right) p_{j}^{\delta_{j}-1}$ values of $x_{j}^{\prime}$ modulo $p_{j}^{\delta_{j}}$ such that $A_{1}+x_{j}^{\prime} A_{2}$ is coprime to $p_{j}$.

Summarizing we have

$$
\begin{aligned}
L & =\varphi\left(B_{3}\right) \varphi\left(A_{1} B_{1}\right) \varphi\left(B_{2}\right) \prod_{i=1}^{r}\left(p_{i}-2\right) p_{i}^{\delta_{i}-1} \\
& =\varphi\left(A_{1} B_{1} B_{2} B_{3}\right) \varphi^{\prime}(D) \\
& =\varphi\left(A_{1} B\right) \varphi^{\prime}(D) .
\end{aligned}
$$

We obtain the following as a corollary of the previous result. 
Lemma 3.6 Let $D$ be the largest divisor of $A$ coprime with $A_{1} A_{2} A_{3}$ and let $B=A / D$. Then $L=S$ if and only if $B \in\{1,2\}$ and $D \in\{1,3\}$.

Proof Let $B=B_{1} B_{2} B_{3}$, where $B_{i}$ are defined in the proof of Lemma 3.5. Then

$$
\begin{aligned}
S & =\frac{\varphi(N)}{\varphi\left(m_{1}\right)} \\
& =\frac{\varphi\left(A_{1} B_{1}\right) \varphi\left(A_{2} B_{2}\right) \varphi\left(A_{3} B_{3}\right) \varphi(D)}{\varphi\left(A_{2} A_{3}\right)} \\
& =\frac{\varphi\left(A_{1} B_{1}\right) \varphi\left(A_{2} B_{2}\right) \varphi\left(A_{3} B_{3}\right) \varphi(D)}{\varphi\left(B_{1}\right) \varphi\left(A_{2} B_{2}\right) \varphi\left(A_{3} B_{3}\right) \varphi(D)} \\
& =\frac{\varphi\left(A_{1} B_{1}\right)}{\varphi\left(B_{1}\right)} .
\end{aligned}
$$

Hence $L=S$ if and only if $\varphi\left(A_{1} B\right) \varphi\left(B_{1}\right) \varphi^{\prime}(D)=\varphi\left(A_{1} B_{1}\right)$. Since, in general, $\varphi\left(A_{1} B\right) \geq$ $\varphi\left(A_{1} B_{1}\right)$, we obtain $\varphi\left(A_{1} B\right)=\varphi\left(A_{1} B_{1}\right)$ and $\varphi\left(B_{1}\right)=\varphi^{\prime}(D)$. This can only happen when $D \in\{1,3\}$ and $B_{1} \in\{1,2\}$. If $B_{1}=2$ then for $B>B_{1}$ we get a contradiction as in this case $\varphi\left(A_{1} B\right)>\varphi\left(A_{1} B_{1}\right)$. So $B=B_{1}=2$. If $B_{1}=1$ then we get $\varphi\left(A_{1} B\right)=\varphi\left(A_{1}\right)$ which means that either $B=1$ or $B=2$ and $A_{1}$ is odd.

Conversely, suppose $D \in\{1,3\}$ and $B_{1} \in\{1,2\}$. Then $L=\varphi\left(A_{1} B\right) \varphi\left(B_{1}\right)$ and $S=$ $\varphi\left(A_{1} B_{1}\right) / \varphi\left(B_{1}\right)$. If $B=1$ then also $B_{1}=1$ and obviously $L=S$. If $B=2$, then either $B_{1}=1$ or $B_{1}=2=B$. In the first case $A_{1}$ is odd and then $L=\varphi\left(A_{1}\right)=S$. In the second case, $L=\varphi\left(2 A_{1}\right)=S$. The lemma is proved.

Lemma 3.7 Let $A, A_{1}, A_{2}, A_{3}$ be the Maclachlan decomposition 2.4 of an admissible signature $\left(m_{1}, m_{2}, m_{3}\right)$ for $N$ with $m_{1}=m_{2}$ and let $S=1$. Then $1<L \leq 6$ if and only if it is given in Table 1 or in Table 2.

Proof Observe that $S=1$ if and only if $m_{1}=N$, as by Sect. 2.3, $N=1 \mathrm{~cm}\left(m_{1}, m_{2}\right)=$ $m_{1}=m_{2}$. The list of all cases for which $L \leq 6$ can be easily derived from the formula (12). First of all, it follows from it that $D \in\{1,3,5,7,9,15,21\}$ (note that $D$ cannot be even). After fixing $D$ we take the condition $\varphi^{\prime}(D) \varphi\left(A_{1} B\right) \leq 6$ to be satisfied.

Let first $m_{3}=N$ and let us list all cases giving a signature $(N, N, N)$ with $1<L \leq 6$. Here we have $A=N, A_{1}=A_{2}=A_{3}=1, D=N, B=1$ and so $1<L \leq 6$ if and only if it is given in Table 1 . Observe here that $L=1$ implies $N=3$.

Now let $m=m_{3}<N$. In this case we have $A_{1}=A_{2}=1$ and so $B_{1}=B_{2}=1$. The only condition on $A_{3}$ is that it must be divisible by primes dividing $B=B_{3}$ if $B>1$, and coprime to $D$. So we have all cases listed in Table 2 .

Table $1 L \leq 6$ for $m_{1}=m_{2}=m_{3}=N$

\begin{tabular}{rllrrrrr}
\hline$A_{1}$ & $A_{2}$ & $A_{3}$ & $A$ & $D$ & $B$ & $L$ & $N$ \\
\hline 1 & 1 & 1 & 5 & 5 & 1 & 3 & 5 \\
1 & 1 & 1 & 7 & 7 & 1 & 5 & 7 \\
1 & 1 & 1 & 9 & 9 & 1 & 3 & 9 \\
1 & 1 & 1 & 15 & 15 & 1 & 3 & 15 \\
1 & 1 & 1 & 21 & 21 & 1 & 5 & 21 \\
\hline
\end{tabular}


Table $2 L \leq 6$ for $m_{1}=m_{2}=N, m_{3}<N$

\begin{tabular}{|c|c|c|c|c|c|c|c|}
\hline$A_{1}$ & $A_{2}$ & $A_{3}$ where $\operatorname{gcd}\left(A_{3}, D\right)=1$ & $A$ & $\mathrm{D}$ & $B$ & $L$ & $N$ divisible by \\
\hline \multirow[t]{14}{*}{1} & 1 & $N / 3, N / 4, N / 6$ & $3,4,6$ & 1 & $3,4,6$ & 2 & $9,8,36$ \\
\hline & & $N / 5, N / 8, N / 10, N / 12$ & $5,8,10,12$ & & $5,8,10,12$ & 4 & $25,16,100,72$ \\
\hline & & $N / 7, N / 9, N / 18$ & $7,9,18$ & & $7,9,18$ & 6 & $49,27,108$ \\
\hline & & $N / 12$ & 12 & 3 & 4 & 2 & 24 \\
\hline & & $N / 15, N / 24, N / 30$ & $15,24,30$ & & $5,8,10$ & 4 & $45,48,300$ \\
\hline & & $N / 21$ & 21 & & 7 & 6 & 147 \\
\hline & & $\mathrm{N} / 5$ & 5 & 5 & 1 & 3 & 5 \\
\hline & & $N / 15, N / 20, N / 30$ & $15,20,30$ & & $3,4,6$ & 6 & $45,40,180$ \\
\hline & & $\mathrm{N} / 7$ & 7 & 7 & 1 & 5 & 7 \\
\hline & & $N / 9, N / 18$ & 9,18 & 9 & 1,2 & 3 & 9,36 \\
\hline & & $N / 36$ & 36 & & 4 & 6 & 72 \\
\hline & & $N / 15, N / 30$ & 15,30 & 15 & 1,2 & 3 & 15,60 \\
\hline & & $N / 60$ & 60 & & 4 & 6 & 120 \\
\hline & & $N / 21, N / 42$ & 21,42 & 21 & 1,2 & 5 & 21,84 \\
\hline
\end{tabular}

Proposition 3.8 If $S=L$ for an admissible signature $\left(m_{1}, m_{2}, m_{3}\right)$ then this signature is rigid. The converse holds for all admissible signatures except for $(5,5,5),(9,9,9)$, $(15,15,15)$, which are rigid and $(S, L)=(1,3)$, and signatures:

$$
\begin{array}{ll}
(N, N, 3), & \text { for } N=9 t, \quad t \in \mathbb{N}, \\
(N, N, 4), & \text { for } N=16 t, t \in \mathbb{N}, \\
(N, N, 6), & \text { for } N=36 t, t \in \mathbb{N}, \\
(N, N, 12), & \text { for } N=48 t, t \in \mathbb{N}, 3 \nmid t
\end{array}
$$

which are rigid and $(S, L)=(1,2)$.

Proof For $L=S$, the rigidity follows from the faithfulness of the action of $\mathcal{S}$ on $\mathcal{L}$, which we proved in the Lemma 3.3. The converse is also true if the $m_{i}$ are pairwise distinct or $L>6$. So assume that $L \leq 6$ and $m_{i}$ are not pairwise distinct. Then $m_{1}$ and $m_{2}$ are equal to $N$, and in particular $S=1$ and the list of all configurations of $N, m_{1}, m_{2}, m_{3}$ are given in Lemma 3.7. The case $m_{3}=N$ is easy; here one can show that $(5,5,5),(9,9,9)$ and $(15,15,15)$ are rigid signatures, while each of the signatures $(7,7,7)$ and $(21,21,21)$ is not rigid since it allows two nonequivalent actions corresponding to $\left(a, a, a^{5}\right),\left(a, a^{2}, a^{4}\right)$ and $\left(a, a, a^{19}\right),\left(a, a^{4}, a^{16}\right)$, respectively.

The case $m_{3}<N$ described in Table 2 is a bit more involved. First, note that there is no rigid action for $L>2$ and so we can assume that $\left(m_{1}, m_{2}, m_{3}\right)=(N, N, 3),(N, N, 4),(N, N, 6)$ or $(N, N, 12)$.

It is easy to show that the signatures $(N, N, 3)$ and $(N, N, 6)$ are rigid. For $(N, N, 3)$, we know $N=9 t$ and we have to consider the actions $\left(a, a^{6 t-1}, a^{3 t}\right)$ and $\left(a, a^{3 t-1}, a^{6} t\right)$ as $a^{3 t}$ and $a^{6 t}$ are the only elements of order 3 in the group $\left\langle a \mid a^{9 t}\right\rangle$. But the function determined by the correspondence $a \mapsto a^{3 t-1}$ moves $a^{6 t-1}$ to $a^{(3 t-1)(6 t-1)}=a$ and $a^{3 t}$ moves to $a^{(3 t-1) 3 t}=a^{-3 t}=a^{6 t}$. Hence these two actions are equivalent. Similarly, for the signature $(N, N, 6)$ we have $N=36 t$ and in the group $\left\langle a \mid a^{36 t}\right\rangle$ we have only two elements of order 6 , namely $a^{6 t}$ and $a^{-6 t}$. So we have to consider the actions $\left(a, a^{30 t-1}, a^{6 t}\right)$ and $\left(a, a^{6 t-1}, a^{-6 t}\right)$ only, which are equivalent by the analogous arguments as for the previous case. 
Let us consider the signature $(N, N, 4), N=8 t$. Similar to the previous cases, we have two actions:

$$
\left(a, a^{2 t-1}, a^{6 t}\right), \quad\left(a, a^{6 t-1}, a^{2 t}\right) .
$$

Now the function induced by the correspondence $a \mapsto a^{6 t-1}$ moves $a^{2 t-1}$ to $a^{(2 t-1)(6 t-1)}=$ $a^{4 t^{2}+1}$. If $t$ is even then this element is equal to $a$ and we have equivalence of both actions. If $t$ is odd then $t^{2} \equiv 1(\bmod 8)$ hence $4 t^{2}+1 \equiv 5(\bmod 8)$, which means that $a^{4 t^{2}+1} \neq a$ and so the actions are not equivalent.

Finally take the signature $(N, N, 12)$, where $N=24 t$. Note that in this case, 3 does not divide $t$ as by Table $2, A_{3}=N / m_{3}$ is coprime to $D=3$. Since, a group of order $24 t$ generated by an element $a$ has exactly four elements of order 12, $a^{2 t}, a^{10 t} a^{14 t}$ and $a^{22 t}$, we have four possibilities for actions:

$$
\left(a, a^{2 t-1}, a^{22 t}\right), \quad\left(a, a^{10 t-1}, a^{14 t}\right), \quad\left(a, a^{14 t-1}, a^{10 t}\right), \quad\left(a, a^{22 t-1}, a^{2 t}\right) .
$$

But only two these are of the type $(N, N, 12)$. In fact, if $t \equiv 1(\bmod 3)$, then $10 t-1,22 t-1 \equiv$ $0(\bmod 3)$ and if $t \equiv 2(\bmod 3)$, then $2 t-1,14 t-1 \equiv 0(\bmod 3)$. Let us consider the first case. The function induced by the correspondence $a \mapsto a^{14 t-1}$ moves $a^{2 t-1}$ to $a^{(2 t-1)(14 t-1)}=a^{4 t^{2}+8 t+1}$. Since $4 t^{2}+8 t+1=4 t(t+2)+1$, we see that only for even $t$ we have $4 t^{2}+8 t+1 \equiv 1(\bmod 24 t)$. Hence, only in this case both actions are equivalent. Let $t \equiv 2(\bmod 3)$ and take the function induced by the correspondence $a \mapsto a^{22 t-1}$. It moves $a^{10 t-1}$ to $a^{(22 t-1)(10 t-1)}=a^{4 t^{2}+16 t+1}$. Again, since $4 t^{2}+16 t+1=4 t(t+4)+1 \equiv 1$ $(\bmod 24 t)$ only for even $t$, the actions $\left(a, a^{10 t-1}, a^{14 t}\right)$ and $\left(a, a^{22 t-1}, a^{2 t}\right)$ are equivalent just for $t$ even which completes the proof.

All of these allow us to deduce our principal result in this paper.

Theorem 3.9 Let $\mathbb{Z}_{N}$ be a cyclic action with signature $\left(m_{1}, m_{2}, m_{3}\right)$ and let $A, A_{1}, A_{2}, A_{3}$ be the Maclachlan decomposition (6) of $\left(m_{1}, m_{2}, m_{3}\right)$. Then the action is weakly rigid if and only if one the following happen

(1) $A=1,2$,

(2) $A=3,6$ and $\operatorname{gcd}\left(A_{1} A_{2} A_{3}, 3\right)=1$,

(3) $A=5,9,15$ and $A_{1}=A_{2}=A_{3}=1$,

(4) $A=3, A_{1}=A_{2}=1$, and $A_{3} \equiv 0(\bmod 3)$,

(5) $A=4, A_{1}=A_{2}=1$, and $A_{3} \equiv 0(\bmod 4)$,

(6) $A=6, A_{1}=A_{2}=1$, and $A_{3} \equiv 0(\bmod 6)$,

(7) $A=12, A_{1}=A_{2}=1, A_{3} \equiv 0(\bmod 4)$, and $\operatorname{gcd}\left(A_{3} / 4,3\right)=1$.

In particular the signature $\left(m_{1}, m_{2}, m_{3}\right)$ is not rigid for $A \notin\{1,2,3,4,5,6,12\}$.

\section{Examples, equations, remarks, and open problems}

The signature $\left(m_{1}, m_{2}, m_{3}\right)$ is said to be $(g, N)$-admissible if there exists a self-homeomorphism $\varphi$ of order $N$ acting on a closed orientable surface $X$ of genus $g$ so that $X / \varphi$ is the sphere ramified over three points with ramification indices $m_{1}, m_{2}, m_{3}$ or equivalently having three singular orbits of the lengths $N / m_{1}, N / m_{2}, N / m_{3}$. 


\subsection{Rigid signature vs rigid order}

Let $X$ be a fixed, closed orientable surface of genus $g \geq 2$. The notion of rigid cyclic action on $X$ leads us to define the concept of rigid order $N$ for $g \geq 2$ and, similarly, the notion of weakly rigid action gives rise to the concept of rigid signature for $g$. This, in turn, allows to define the concept of weakly rigid order which means that all admissible signatures are rigid. In this subsection we shall consider cyclic actions with the orbit genus zero and having three singular orbits of the lengths $m_{1}, m_{2}, m_{3}$, calling such actions quasi-platonic. Let $N_{1}, \ldots, N_{k}$ be all possible orders of all such actions on $X$. Now $N=N_{i}$ can fail to be rigid order for two reasons. The first is that there may exist few distinct admissible signatures and, in principle, some of them may be rigid and the others not. The second, more subtle reason for nonrigidity of $N$ for genus $g$, is that although there may exist just one admissible signature, this signature is not rigid. The previously mentioned results of Nakagawa and Hirose imply that the cyclic actions of orders $4 g+1,4 g, 3 g+3,3 g$ on closed orientable surfaces of genus $g$, where $g \geq 12$ are rigid. Additionally, if $g \not \equiv 2(\bmod 3)$, then $N$ is also rigid. All of these phenomena, which show that rigidity of cyclic actions is essentially coarser than week rigidity, are well illustrated in Tables 3 and 4.

\subsection{Cyclic actions with fixed-points free self-homeomorphisms}

The pair $(g, N)=(11,30)$ in Table 4 is an interesting case because these are the smallest $g$ and $N$ values for which there exists an action with fixed-point free acting selfhomeomorphism. There are many more cyclic actions containing fixed point free acting self-homeomorphisms. The other two examples are $(g, N)=(16,42)$ or $(25,60)$ with signatures $(21,14,6)$ and $(20,15,12)$, respectively.

Another interesting case is $N=210$ in which $\mathbb{Z}_{N}$ can act on surfaces of three consecutive genera, $g=95,96$, and 97 . The corresponding signatures are $(70,42,15),(70,30,21)$, and $(42,35,30)$, respectively, all of which are rigid since $A=1$ in each case. Additionally, there are no other 210-admissible signatures for $g=95$, so 210 is a rigid order for $g=95$. For $g=96$, we have one more 210-admissible signature, $(210,42,15)$, which gives rigid action. In fact, let $G$ be a cyclic group of order 210 and let us represent elements of $G$ as 4-tuples of elements of the direct product $G=\left\langle a_{2}\right\rangle \times\left\langle a_{3}\right\rangle \times\left\langle a_{5}\right\rangle \times\left\langle a_{7}\right\rangle$, where $a_{i}$ has order $i$. Now for a fixed element $x$ of order 210 , say $x=\left(a_{2}, a_{3}, a_{5}, a_{7}\right)$ we have exactly one element $y$ of order 42 such that $x y$ has order 15 , namely $y=\left(a_{2}, a_{3}, 1, a_{7}^{-1}\right)$. So we see that $N=210$ is a weakly rigid order for $g=96$. Finally for $g=97$, we have three more 210 -admissible signatures $(210,30,21),(210,70,15)$, and $(210,105,14)$ whose Maclachlan decomposition are respectively $(3,1,7,10),(5,1,3,14)$, and $(7,1,2,15)$. The first case defines rigid action, as for $x=\left(a_{2}, a_{3}, a_{5}, a_{7}\right)$ again we have exactly one element $y=\left(a_{2}, a_{3}, a_{5}^{-1}, 1\right)$ of order 30 such that $x y$ has order 21 . The last two cases are not rigid. In the case $(210,70,15)$, for $x=\left(a_{2}, a_{3}, a_{5}, a_{7}\right)$, we have exactly three elements $y$ of order 70 such that $x y$ has order 15 , namely $y=\left(a_{2}, 1, a_{5}^{k}, a_{7}^{-1}\right), k=1,2,3$. In the case $(210,105,14)$, for $x=\left(a_{2}, a_{3}, a_{5}, a_{7}\right)$, we have exactly five elements $y$ of order 105 such that $x y$ has order 14 , namely $y=\left(1, a_{3}^{-1}, a_{5}^{-1}, a_{7}^{k}\right), k=1,2,3,4,5$. So for $g=97$, $N=210$ is not a weakly rigid order. So, all three phenomena: rigidity, weak rigidity and not weak rigidity can occur for cyclic action, allowing fixed point free self-homeomorphisms for the same order, on surfaces of three consecutive genera. 
Table 3 Rigidity of quasi-platonic cyclic actions on surfaces of genera $2 \leq g \leq 7$

\begin{tabular}{|c|c|c|c|c|}
\hline Genus & Order & Signature & Rigidity of order & Rigidity of signature \\
\hline \multirow[t]{4}{*}{2} & 10 & $(10,5,2)$ & Rigid & Rigid \\
\hline & 8 & $(8,8,2)$ & Rigid & Rigid \\
\hline & 6 & $(6,6,3)$ & Rigid & Rigid \\
\hline & 5 & $(5,5,5)$ & Rigid & Rigid \\
\hline \multirow[t]{6}{*}{3} & 14 & $(14,7,2)$ & Rigid & Rigid \\
\hline & 12 & $(12,4,3)$ & Weakly-rigid & Rigid \\
\hline & & $(12,12,2)$ & & Rigid \\
\hline & 9 & $(9,9,3)$ & Rigid & Rigid \\
\hline & 8 & $(8,8,4)$ & Non-rigid & Non-rigid \\
\hline & 7 & $(7,7,7)$ & Non-rigid & Non-rigid \\
\hline \multirow[t]{7}{*}{4} & 18 & $(18,9,2)$ & Rigid & Rigid \\
\hline & 16 & $(16,16,2)$ & Rigid & Rigid \\
\hline & 15 & $(15,5,3)$ & Rigid & Rigid \\
\hline & 12 & $(12,6,4)$ & Weakly-rigid & Rigid \\
\hline & & $(12,12,3)$ & & Rigid \\
\hline & 10 & $(10,10,5)$ & Non-rigid & Non-rigid \\
\hline & 9 & $(9,9,9)$ & Rigid & Rigid \\
\hline \multirow[t]{5}{*}{5} & 22 & $(22,11,2)$ & Rigid & Rigid \\
\hline & 20 & $(20,20,2)$ & Rigid & Rigid \\
\hline & 15 & $(15,15,3)$ & Rigid & Rigid \\
\hline & 12 & $(12,12,6)$ & Rigid & Rigid \\
\hline & 11 & $(11,11,11)$ & Non-rigid & Non-rigid \\
\hline \multirow[t]{9}{*}{6} & 26 & $(26,13,2)$ & Rigid & Rigid \\
\hline & 24 & $(24,24,2)$ & Rigid & Rigid \\
\hline & 21 & $(21,7,3)$ & Rigid & Rigid \\
\hline & 20 & $(20,5,4)$ & Rigid & Rigid \\
\hline & 18 & $(18,18,3)$ & Rigid & Rigid \\
\hline & 16 & $(16,16,4)$ & Rigid & Rigid \\
\hline & 15 & $(15,15,5)$ & Non-rigid & Non-rigid \\
\hline & 14 & $(14,14,7)$ & Non-rigid & Non-rigid \\
\hline & 13 & $(13,13,13)$ & Non-rigid & Non-rigid \\
\hline \multirow[t]{8}{*}{7} & 30 & $(30,15,2)$ & Rigid & Rigid \\
\hline & 28 & $(28,28,2)$ & Rigid & Rigid \\
\hline & 24 & $(24,8,3)$ & Rigid & Rigid \\
\hline & 21 & $(21,21,3)$ & Rigid & Rigid \\
\hline & 20 & $(20,10,4)$ & Rigid & Rigid \\
\hline & 18 & $(18,9,6)$ & Non-rigid & Non-rigid \\
\hline & 16 & $(16,16,8)$ & Non-rigid & Non-rigid \\
\hline & 15 & $(15,15,15)$ & Rigid & Rigid \\
\hline
\end{tabular}


Table 4 Rigidity of quasi-platonic cyclic actions on surfaces of genera $8 \leq g \leq 11$

\begin{tabular}{|c|c|c|c|c|}
\hline Genus & Order & Signature & Rigidity of order & Rigidity of signature \\
\hline \multirow[t]{6}{*}{8} & 34 & $(34,17,2)$ & Rigid & Rigid \\
\hline & 32 & $(32,32,2)$ & Rigid & Rigid \\
\hline & 24 & $(24,24,3)$ & Rigid & Rigid \\
\hline & 20 & $(20,20,5)$ & Non-rigid & Non-rigid \\
\hline & 18 & $(18,18,9)$ & Non-rigid & Non-rigid \\
\hline & 17 & $(17,17,17)$ & Non-rigid & Non-rigid \\
\hline \multirow[t]{10}{*}{9} & 38 & $(38,19,2)$ & Rigid & Rigid \\
\hline & 36 & $(36,36,2)$ & Rigid & Rigid \\
\hline & 30 & $(30,10,3)$ & Rigid & Rigid \\
\hline & 28 & $(28,7,4)$ & Rigid & Rigid \\
\hline & 27 & $(27,27,3)$ & Rigid & Rigid \\
\hline & 24 & $(24,8,6)$ & Non-rigid & Rigid \\
\hline & & $(24,24,4)$ & & Non-rigid \\
\hline & 21 & $(21,21,7)$ & Non-rigid & Non-rigid \\
\hline & 20 & $(20,20,10)$ & Non-rigid & Non-rigid \\
\hline & 19 & $(19,19,19)$ & Non-rigid & Non-rigid \\
\hline \multirow[t]{11}{*}{10} & 42 & $(42,21,2)$ & Rigid & Rigid \\
\hline & 40 & $(40,40,2)$ & Rigid & Rigid \\
\hline & 33 & $(33,11,3)$ & Rigid & Rigid \\
\hline & 30 & $(30,6,5)$ & Weakly-rigid & Rigid \\
\hline & & $(30,30,3)$ & & Rigid \\
\hline & 28 & $(28,14,4)$ & Rigid & Rigid \\
\hline & 25 & $(25,25,5)$ & Non-rigid & Non-rigid \\
\hline & 24 & $(24,24,6)$ & Non-rigid & Rigid \\
\hline & & $(24,12,8)$ & & Non-rigid \\
\hline & 22 & $(22,22,11)$ & Non-rigid & Non-rigid \\
\hline & 21 & $(21,21,21)$ & Non-rigid & Non-rigid \\
\hline \multirow[t]{6}{*}{11} & 46 & $(46,23,2)$ & Rigid & Rigid \\
\hline & 44 & $(44,44,2)$ & Rigid & Rigid \\
\hline & 33 & $(33,33,3)$ & Rigid & Rigid \\
\hline & 30 & $(15,10,6)$ & Rigid & Rigid \\
\hline & 24 & $(24,24,12)$ & Non-rigid & Non-rigid \\
\hline & 23 & $(23,23,23)$ & Non-rigid & Non-rigid \\
\hline
\end{tabular}

\subsection{Infinite series of non-rigidity examples}

Example 1 Let $p$ be an odd prime and let $\mathbb{Z}_{p^{n}}$ be a quasi-platonic action on a closed orientable surface $X=X_{g}$ corresponding to a signature $\left(m_{1}, m_{2}, m_{3}\right)$, where $m_{1} \geq m_{2} \geq m_{3}$. By Theorem 3.9, this action is not rigid for $p>7$ or $p=5$ with $n>1$. By (5), $m_{1}=m_{2}=p^{n}$ and $m_{3}=p^{m}$ for some $m \leq n$. We can find explicit examples of non-conjugated actions. Let us first assume $m=n$. Then

$$
\left(a, a, a^{-2}\right) \text { and }\left(a, a^{2}, a^{-3}\right)
$$


are non-equivalent under the action of $\mathbb{Z}_{p^{n}}^{*} \rtimes S_{3}$. These two signatures correspond to the algebraic curves

$$
y^{p^{n}}=x(x-1) \text { and } y^{p^{n}}=x(x-1)^{2} .
$$

Now let $m<n$. Then for arbitrary $q<p,\left(a, a^{q p^{n}-1}, a^{q p^{n}}\right)$ is a defining signature. For $q=1$ and $q$ not satisfying the congruence

$$
\left(p^{n-m}-1\right)\left(q p^{n-m}=1\right) \equiv 1 \quad\left(\bmod p^{n}\right),
$$

the corresponding curves given by

$$
y^{p^{n}}=x(x-1)^{q p^{n}-1}
$$

are not isomorphic.

Example 2 Let $p, q$ be distinct odd primes with $p<q$, and let $\mathbb{Z}_{p q}$ act on a surface $X$ defined by an admissible signature $\left(m_{1}, m_{2}, m_{3}\right)$. Then for $\left(m_{1}, m_{2}, m_{3}\right)=(p, q, p q)$ the action is rigid while for $\left(m_{1}, m_{2}, m_{3}\right)=(p q, p q, p q)$ we have two generating signatures of elements from $\mathbb{Z}_{p q}$,

$$
\left(a, a, a^{-2}\right) \text { and }\left(a, a^{2}, a^{-3}\right) \text {, }
$$

which define non-equivalent actions for $p \geq 5$. By direct calculations we obtain non-rigidity for $p=3$ except for $q=5$ and $q=7$. These two signatures correspond to the algebraic curves

$$
C_{1}:=y^{p q}=x(x-1) \text { and } C_{2}:=y^{p q}=x(x-1)^{2} .
$$

\subsection{Conformal rigidity of weakly rigid actions}

Observe that a triangle in the hyperbolic plane is determined, up to isometry, by its angles and so a Fuchsian group with signature $(0 ; k, l, m)$ is unique up to conjugation by an isometry. This means that a rigid signature determines uniquely the structure described in Sect.2.1. In particular, for a rigid order $N$, there is just one conformal structure on $X_{g}$ for which $\mathbb{Z}_{N}$ is the group of its conformal automorphisms. So the topological rigidity implies conformal rigidity.

\subsection{Real forms}

Observe that all quasi-platonic surfaces with cyclic actions are symmetric, due to the result of Singerman [9], since the map $a \mapsto a^{-1}, b \mapsto b^{-1}$ induces an automorphism of any abelian group generated by $a$ and $b$. The topological types of the corresponding real forms have been found in [3]. If all periods corresponding to the action are distinct then $\mathbb{Z}_{N}$ is the full group of automorphisms of the surface $X$ and it has one or two distinct real forms, according to $N$, being odd or even, respectively. In addition these forms are both connected and nonseparable. If some of periods are equal, say $a$ and $b$ have the same order, then the situation is more involved, because there is another automorphism of $\mathbb{Z}_{N}$ induced by the map $a \mapsto b^{-1}, b \mapsto a^{-1}$. This implies that $\mathbb{Z}_{N}$ is no longer the full group of automorphisms of $X$, so $X$ may have 2,3 , or 4 distinct real forms. They can be both separable or not and connected or not, or even purely imaginary. 


\subsection{Open problems}

(1) In [3], the authors give equations of the corresponding surfaces $X$, and explicit formulas for the generators of $\mathrm{Aut}^{ \pm}(X)$. It could be interesting to find defining equations for the corresponding real forms, at least for the rigid signatures described in this paper.

(2) Find sharp bounds for the number $R(g)$ of rigid and $W R(g)$ of weakly rigid actions on a closed orientable surface of genus $g \geq 2$.

(3) Investigate asymptotic behavior of the ratios $A(g) / R(g), A(g) / W R(g)$ and $R(g) / A(g)$, $W R(g) / A(g)$, where $A(g)$ denotes the number of all quasi-platonic actions.

Acknowledgments The authors are grateful to Dr. B. Szepietowski and to Prof. X. Zhao for their useful remarks. They also would like to thank the referee for carefully reading the paper, many helpful suggestions and, after all, for pointing us a gap in Lemma 3.7 and in its proof.

Open Access This article is distributed under the terms of the Creative Commons Attribution 4.0 International License (http://creativecommons.org/licenses/by/4.0/), which permits unrestricted use, distribution, and reproduction in any medium, provided you give appropriate credit to the original author(s) and the source, provide a link to the Creative Commons license, and indicate if changes were made.

\section{References}

1. Belyi, G.: On Galois extensions of a maximal cyclotomic field. Math. USSR Izv. 14(2), 247-256 (1980)

2. Benim, R., Wootton, A.: Enumerating quasi-platonic cyclic group actions. Rocky Mount. J. Math. 43(5), 1459-1480 (2013)

3. Bujalance, E., Cirre, J.F., Turbek, P.: Symmetry types of cyclic covers of the sphere. Israel J. Math. 191(1), 61-83 (2012)

4. Hidalgo, R.A.: Homology closed Riemann surfaces. Q. J. Math. 63, 931-952 (2012)

5. Hirose, S.: On periodic maps over surfaces with large periods. Tohoku Math. J. 62(1), 45-53 (2010)

6. Köck, B., Singerman, D.: Real Belyi theory. Q. J. Math. 58, 463-478 (2007)

7. Nakagawa, K.: On the orders of automorphisms of a closed Riemann surface. Pac. J. Math. 115, 435-443 (1984)

8. Nielsen, J.: Die Struktur periodischer Transformationen von Flächen. Math. Fys. Medd. Danske Vid. Selsk 15 (1) (1937) (English transl. in Jakob Nielsen collected works, 2, 65-102)

9. Singerman, D.: Symmetries of Riemann surfaces with large automorphism group. Math. Ann. 210, 17-32 (1974)

10. Wootton, A.: Defining equations for cyclic prime covers of the Riemann sphere. Israel J. Math. 157, 103-122 (2007)

11. Yokoyama, K.: Classification of periodic maps on compact surfaces. I. Tokyo J. Math. 6, $75-94$ (1983) 